\title{
Exportação a Mercados Internacionais com Graus Diferen- tes de Desenvolvimento: um estudo com microdados de firmas argentinas no período recente
}

\section{Eva Yamila da Silva Catela}

Professora - Universidade Federal de Santa Catarina

Endereço: Rua Eng. Agronômico Andrei Cristian Ferreira, s/n, Centro Socioeconômico, Bloco D, $2^{\circ}$ Andar, sala 205 - Trindade - Florianópolis/SC - CEP: 88040-900 - E-mail: eva.yamila@ufsc.br https://orcid.org/0000-0002-5973-3116

\section{Dimitri da Costa Bessa}

Pesquisador - Secretaria de Estado do Planejamento de Santa Catarina (SPG/SC) Endereço: Rod. SC 401, Km 5, no 4600 - Centro Administrativo - Bloco 3, $2^{\circ}$ andar - Florianópolis/SC CEP: 88032-000 - E-mail: dimitribessa@gmail.com - https://orcid.org/0000-0002-9937-8219

Recebido: 22/04/2016. Aceite: 30/10/2017.

\section{Resumo}

O objetivo deste trabalho é analisar a penetração de empresas argentinas da indústria de transformação a mercados externos com diferentes graus de desenvolvimento. Para isto, utiliza-se a base de dados ENDEI, onde constam informações de emprego e atividades de inovação através de uma amostra estratificada (setor e tamanho) de 3.691 firmas, constituindo a base de dados de comércio exterior da Aduana Argentina. Dois modelos são estimados: um modelo probit ordenado, em que a ordenação se associa ao número e complexidade dos mercados nos quais as empresas atuam e um modelo de diversificação da exportação, considerando a distribuição de Poisson para o número de produtos exportados. Os resultados apontam que empresas que exportam para mercados de maior complexidade ou têm uma pauta exportadora mais diversificada, em termo de produtos, apresentam produção mais intensiva em tecnologia e em trabalho qualificado, participam ativamente de redes de cooperação com instituições públicas e privadas e importam insumos e novas tecnologias de outros países.

\section{Palavras-Chave}

Competitividade internacional. Microdados. Argentina.

\begin{abstract}
The purpose of this paper is to analyze the entry of Argentinian industry firms to external markets with different degrees of development. For this, the ENDEl database is used, which has employment and innovation activities information, of a sector and size stratified sample of 3,691 firms, as well as the foreign trade database of Argentinian Customs. Two models are estimated: an ordered probit model, in which ordering is associated with the number and complexity of markets in which firms operate and a model of export diversification, considering Poisson distribution for the number of exported products. The results show that firms that export to markets of greater complexity or have a more diversified export in terms of products,
\end{abstract}


show more intensive production in technology and skilled labor, are more strongly involved in cooperation networks with public and private institutions and import inputs and new technologies from other countries.

\section{Keywords}

International competitiveness. Microdata. Argentina.

\section{JEL Classification}

D22. F14. F19. L25. O32.

\section{Introdução}

A pesquisa teórica e empírica em comércio internacional destaca a importância de serem consideradas qualificações, decisões e comportamento de empresas para entender o fluxo do comércio entre países. A natureza global dos mercados e atividades produtivas e econômicas resultou em aumento da concorrência e em uma nova e crescente divisão do trabalho, impondo mudanças nas estratégias das empresas.

Tamanho, produtividade e outras características das empresas (Melitz e Redding 2014), bem como a inovação em um contexto de complexidade tecnológica, são fundamentais na determinação dos custos unitários e, consequentemente, do desempenho no comércio internacional (Antonelli 2008; Dosi et al. 2015), tornando a competitividade mais dependente de estratégias e estrutura do que de características do país (Aiginger et al. 2015).

Estudos recentes mostram que os microfundamentos que moldam o padrão de inserção no comércio das empresas vão além das fontes tradicionais, como custos e posição tecnológica relativa no setor produtivo, incluindo a interação em redes de empresas e instituições públicas (Alvarez et al. 2009; Antonelli 2008), a composição da força de trabalho (Itskhoki e Helpman 2015; Fieler et al. 2014), bem como a rede de fornecedores de insumos intermediários (Acemoglu et al. 2007; Bernard et al. 2015).

O objetivo deste trabalho é explorar a influência destes aspectos na penetração da indústria de transformação da Argentina em mercados internacionais com diferentes graus de desenvolvimento. Para isso, estima-se 
um modelo probit ordenado, em que a ordenação se associa ao número e complexidade dos mercados nos quais as empresas atuam. Os dados provêm de duas fontes: (i) a Encuesta Nacional de Dinámica de Empleo e Innovación (ENDEI), ${ }^{1}$ que fornece informações de uma amostra estratificada, por setor e tamanho, de 3691 empresas de 26 setores da indústria de transformação argentina para o período 2010-2012, representativas de empresas com 10 ou mais trabalhadores; e (ii) registros administrativos da Aduana Argentina para comércio exterior, relacionados ao valor exportado, produtos e destinos.

Segundo os registros da ENDEI e da Aduana para o período considerado, o padrão comercial da Argentina distingue-se por três características: i) menos de $25 \%$ das empresas argentinas exportam; ii) as exportações concentram-se em setores de baixo conteúdo tecnológico; e iii) os principais destinos de exportação são países do Mercosul e América Latina (comércio Sul-Sul). Trabalhos empíricos recentes mostram que as exportações industriais argentinas no período 1998-2011 tiveram um dinamismo importante, que se refletiu tanto no incremento do valor bruto das exportações, como na ampliação da quantidade de indústrias exportadoras (Albornoz et al. 2012; Artopoulos et al. 2013; Albornoz et al. 2014).

Este artigo está dividido em seis seções, incluindo a introdução e conclusões. Na seção 2 são analisados os microfundamentos, que não se restringem a custos dos fatores e inovação tecnológica, do acesso de empresas de um país tecnologicamente distante da fronteira mundial a mercados de exportação. A seção 3 apresenta os dados da pesquisa e algumas evidências empíricas do desempenho das indústrias argentinas no mercado mundial. $\mathrm{Na}$ seção 4 são formuladas hipóteses de trabalho relacionadas à quantidade e qualidade de mercados nos quais uma empresa investe. Os resultados dos modelos propostos são apresentados na seção 5. Os comentários finais estão reservados para a seção 6 .

1 Trata-se de uma pesquisa conjunta do Ministério de Ciência, Tecnologia e Inovação Produtiva, do Ministério do Trabalho, Emprego e Seguridade Social da Argentina, e do Banco Interamericano de Desenvolvimento (BID). 


\section{Microfundamentos do Acesso a Mercados Internacionais e Hipóte- ses de Trabalho}

O objetivo deste trabalho é analisar a relação entre aptidão e características das empresas, de um lado, e acesso a mercados com diferentes graus de desenvolvimento, de outro. Captar a complexidade dos mercados de exportação exige ir além da dicotomia exportar/não exportar e distinguir o acesso a diferentes mercados externos. Para isto, foram criadas quatro categorias de alcance geográfico das exportações das empresas e da quantidade de mercados em que operam: (1) só mercado doméstico; (2) Mercosul e América Latina; (3) África, Oceania e Ásia; ${ }^{2}$ e (4) Europa e América do Norte. Cabe destacar que as categorias superiores podem incluir as inferiores, por exemplo, uma empresa pode estar classificada na categoria (4) e vender também no mercado doméstico e nos mercados das categorias (2) e (3).

A tendência é que quanto maior, mais diversificado e complexo o alcance geográfico da empresa, maior será seu potencial de inserção externa. Assim, a ordenação tenta captar a "qualidade dos mercados" (Bastos e Silva 2010; Hallak 2006), considerando que o acesso a mercados culturalmente distintos e/ou com maior desenvolvimento econômico e tecnológico exigiria maior competência da empresa.

A decisão de explorar mercados estrangeiros depende das capacidades que podem ser associadas a cinco grupos de variáveis: tecnologia, custos, composição da força de trabalho, vinculação institucional a redes e tipo de provedores de insumos. Tradicionalmente, os custos da empresa, em especial o custo laboral, são considerados como um dos determinantes dos preços dos bens e da capacidade de exportação. Trabalhos como BeiseZee e Rammer (2006), Aw et al. (2007), Klein et al. (2013) e Dosi et al. (2015) estimam a relação entre custos de trabalho e atividade exportadora, encontrando uma relação significativa que pode ser negativa ou positiva, dependendo do setor e da intensidade tecnológica dos bens produzidos e das habilidades dos trabalhadores. A atividade de exportação pode aumentar a demanda de trabalhadores qualificados, ensejando um prêmio salarial ao trabalho qualificado e elevando a produtividade. Esta relação entre comércio e qualificação da força laboral tem sido estudada em diferentes contextos (Biscourp e Kramarz 2007; Serti e Tomasi 2014; Iodice e Tomasi

2 A terceira categoria é dominada basicamente por Ásia, dado que são poucas as firmas que exportam bens manufaturados para África e Oceania. 
2015). Há, ainda, um efeito heterogêneo entre categorias ocupacionais, especialmente em países com estruturas exportadoras baseadas em commodities ou manufaturas de baixa intensidade tecnológica, como no caso de países em desenvolvimento (Hummels et al. 2014; Carluccio et al. 2015).

Helpman et al. (2017) mostram que a heterogeneidade da força de trabalho ocorre principalmente entre empresas do mesmo setor. Fieler et al. (2014) apresentam evidências de relação entre maior abertura e aumento da demanda por trabalhadores qualificados na indústria de transformação e, consequentemente, o prêmio por habilidade.

Esses estudos sugerem, portanto, as seguintes hipóteses:

A probabilidade de uma empresa de um país em desenvolvimento, tecnologicamente retardatário, conquistar mercados do próprio país ou de uma nação limítrofe, geograficamente próxima, pertencente à mesma União Aduaneira, ou com cultura similar, é maior que a de penetrar em mercados de países tecnologicamente desenvolvidos, com cultura diferente e mais distantes (H1);

Ascender a mercados mais desenvolvidos demanda, em geral, bens manufaturados de maior qualidade, cuja produção é intensiva em mão de obra qualificada. (H2);

Empresas que não se inserem em atividades inovativas apresentam menor probabilidade de exportar para mercados mais dinâmicos (H3).

O hiato tecnológico do país (Amendola et al. 1993; Guerreri e Meliciani 2005) e as características setoriais (Amable e Verspagen 1995; Laursen e Meliciani 2002; 2010; Dosi et al. 2015) influenciam a relação entre tecnologia e desempenho exportador. Todavia, dependendo do engajamento individual das empresas em atividades inovativas, esta brecha pode ser menor, levando à seguinte hipótese:

O desempenho das empresas melhora com a participação em redes de cooperação que capacitam os trabalhadores, promovem atividades de pesquisa $e$ desenvolvimento (P\&D), desenho industrial e intercâmbio tecnológico, compartilham custos e instalações para realizar provas e ensaios, monitoram a qualidade dos produtos ou patrocinam mudanças organizacionais ${ }^{3}$ (H4).

3 Todas estas atividades são consideradas na Encuesta Nacional de Dinámica de Empleo e Innovación. 
Como documentam Alvarez et al. (2009) e Antonelli (2008), estes ganhos estão associados à capacidade de penetrar em mercados mais desenvolvidos. A vinculação e cooperação para realizar estas atividades são efetuadas por diversos tipos de instituições: empresas do mesmo grupo ou matriz, outras firmas, universidades públicas ou particulares, instituições públicas de ciência e tecnologia, consultores e programas públicos. As redes de cooperação podem envolver também a relação com fornecedores, cuja exportação de bens se associa à importação de insumos intermediários por diversos canais (Gereffi 2014; Antràs et al. 2017; Bernard et al. 2015): redução de custos de produção e acesso a novas variedades ou maior qualidade de insumos. Especificamente para os países em desenvolvimento, há ainda a possibilidade de difusão de tecnologias modernas incorporadas nos insumos intermediários (Antonelli 2008).

Os impactos destes fatores são heterogêneos entre os setores industriais, pois apresentam distintos fluxos de conhecimento, dinâmica de inovação, tamanho, custos e demanda de bens. Por outro lado, para estimar os efeitos específicos das variáveis associadas ao objetivo principal deste trabalho, deve ser feito o controle das características inerentes à empresa que determinam um comportamento diferenciado (Bernard et al. 2012), como tamanho, filiação a um grupo ou conglomerado, participação estrangeira na propriedade e controle do capital (Dunning 2016), e existência de controle familiar. Controlar por estas características reduz o viés de estimação dos coeficientes das variáveis-chave do modelo consideradas nas hipóteses.

\section{Modelos Econométricos}

Emprega-se um modelo probit de probabilidades cumulativas de resposta ordenada (McCullagh 1980; Greene e Hensher 2010). Formalmente, a variável dependente Y é ordenada em J distintas possibilidades (assumindo os valores $1,2 \ldots \mathrm{J}$ ), sendo $\mathrm{j}+\mathrm{l}$ superior a $\mathrm{j}$. O modelo de escolha ordenado é derivado a partir de um modelo de variável latente (que é a utilidade $\mathrm{y}^{*}$ ), determinada por:

$$
y^{*}=x \beta+\epsilon
$$


em que $\mathrm{x}$ é o vetor de covariadas de tamanho lxk e $\beta$ é o vetor de parâmetros desconhecidos com dimensão kxl. As diferentes J escolhas se relacionam com a variável latente considerando pontos de corte $u_{j}$ (ou threshold):

$$
y=j \text { se } u_{j-1} \leq y^{*} \leq u_{j}
$$

A probabilidade condicional de se observar a j-ésima categoria é:

$$
P(y=j \mid x)=P\left(u_{j-1} \leq y^{*} \leq u_{j}\right)=P\left(u_{j-1} \leq x \beta+\epsilon \leq u_{j}\right)
$$

Utiliza-se o procedimento em dois estágios de Heckman (1979) para corrigir o viés de seleção em mercados exportadores das empresas mais produtivas. O modelo de Heckman (Modelo 1), que descreve o controle da margem extensiva do comércio (Helpman et al. 2008), serve de base para testar a estabilidade dos coeficientes do probit ordenado (Modelo lb) e controlar o efeito de seleção nos mercados exportadores ( $\operatorname{Exp}=1$; No Exp=1), considerando a produtividade como o determinante principal da participação em mercados externos (Bernard et al. 2012; Bernard et al. 2015), além de algumas características que também podem influenciar esta decisão, como pertencer a um grupo de empresas (grupo), ter participação estrangeira na propriedade do capital da empresa (cap_internacional), ou ser familiar (empresa_familiar):

$$
\begin{aligned}
& P(\operatorname{Exp}=1 ; \text { NoExp }=0) \\
& \quad=\theta \text { grupo } \alpha \text { cap }_{\text {internacional }}+\text { Bempresa }_{\text {familiar }} \\
& \quad+\text { rproductividad }_{i t-1}+\epsilon_{i t}
\end{aligned}
$$

Assim, a estimativa de $\gamma$ é considerada a restrição de exclusão em mercados exportadores (empresas mais produtivas se autosselecionam para participar de mercados exportadores). Selecionada a participação em mercados externos, o segundo modelo busca os determinantes da qualidade de inserção por meio de duas abordagens. A primeira relaciona-se aos diferentes alcances de mercados:

(Mercadodoméstico $=1 ;$ MercosuryAméricaLatina $=2 ;$ África, OceaniayÁsia

$=3 ;$ EuropayEstadosUnidos $=4$ )

$=\theta$ var. control $_{i t}+\alpha$ inova $_{i t}+\beta$ salario $_{i t-1}+$ sinversión $_{i t-1}$

$+\gamma$ inversa_mills $+\epsilon_{\text {it }}$ 
Neste caso, a razão inversa de Mills resulta da estimação do primeiro modelo e é empregada como variável explicativa na regressão principal de modo a atenuar o viés de seleção amostral.

A segunda abordagem focaliza a dimensão da atividade exportadora pela ótica do número de produtos que a empresa vende nos mercados externos:

$$
\begin{gathered}
P(\text { número de productos })=\theta \text { var.control } \\
\text { it } \\
\text { Sinversió } n_{i t-1}+\gamma \text { inova }_{i t}+\beta \text { salario }_{i t-1}+
\end{gathered}
$$

Desta forma, três modelos são estimados. No modelo 1, a probabilidade de exportar depende basicamente da produtividade, da participação do capital internacional na empresa, da dummy que determina se a empresa forma parte de um conglomerado e/ou se é uma empresa familiar. No modelo 2, a variável dependente é a probabilidade de exportar bens para mercados de qualidades distintas relativamente ao mercado doméstico: Mercosul e América Latina (AL), África, Oceania e Ásia, e Europa e Estados Unidos (E_USA). Por último, a Equação (3) estima a margem intensiva intra-empresa, considerando o número de produtos que exporta (utiliza-se a classificação de seis dígitos da Nomenclatura Comum do Mercosul).

Incluem-se nestes modelos diferentes fatores associados à inserção externa da empresa tratados na seção anterior: variáveis associadas à composição do trabalho e variáveis associadas à participação da empresa em redes com instituições públicas e privadas. Por fim, para tentar captar a existência de um prêmio pela importação de insumos, utilizam-se os tipos de provedores por origem geográfica, sendo os insumos domésticos a categoria de referência.

\section{Fontes de Dados e Estatísticas Descritivas}

Com o intuito de investigar as variáveis associadas à capacidade das empresas de se inserirem em mercados internacionais com diferentes estágios de desenvolvimento, utilizou-se a base de dados Encuesta Nacional de Dinámica de Empleo e Innovación (ENDEI), desenvolvida pelo Ministerio de Trabajo, Empleo y Seguridad Social (MTEySS), em parceria com o 
Ministerio de Ciencia, Tecnología e Innovación Productiva da Argentina. A pesquisa cobre 3691 empresas, com 10 ou mais trabalhadores, da indústria de transformação, de um universo de pouco menos de 18.000 empresas de todo o país A amostra representa $29 \%$ da indústria manufatureira argentina, que concentra $88 \%$ do emprego formal industrial registrado (ENDEI, 2016). A pesquisa refere-se ao período 2010-2012.

Tabela 1 - Distribuição das empresas por setor industrial - 2012

\begin{tabular}{|l|c|c|c|c|}
\hline \multicolumn{1}{|c|}{ Setor da indústria } & Firmas & $\%$ firmas exportadoras & Vendas & Emprego \\
\hline Alimentos, bebidas e tabaco & $21 \%$ & $23 \%$ & $38 \%$ & $32 \%$ \\
\hline Indústria têxtil e do vestuário & $11 \%$ & $20 \%$ & $4 \%$ & $9 \%$ \\
\hline Couro e calçados & $4 \%$ & $23 \%$ & $2 \%$ & $4 \%$ \\
\hline Madeira e móveis & $9 \%$ & $16 \%$ & $2 \%$ & $4 \%$ \\
\hline Papel e edições & $7 \%$ & $25 \%$ & $5 \%$ & $6 \%$ \\
\hline Química e petroquímica & $5 \%$ & $55 \%$ & $10 \%$ & $6 \%$ \\
\hline Farmacêutica & $2 \%$ & $65 \%$ & $4 \%$ & $4 \%$ \\
\hline Borracha e plástico & $6 \%$ & $39 \%$ & $3 \%$ & $5 \%$ \\
\hline Siderurgia e metalurgia & $15 \%$ & $30 \%$ & $10 \%$ & $10 \%$ \\
\hline Máquinas e equipamentos & $7 \%$ & $52 \%$ & $4 \%$ & $5 \%$ \\
\hline Material elétrico, aparelhos elétricos, rádio e televisão. & $3 \%$ & $40 \%$ & $3 \%$ & $3 \%$ \\
\hline Automotivo, construção naval e equipamento ferroviário. & $5 \%$ & $40 \%$ & $12 \%$ & $8 \%$ \\
\hline Outras indústrias & $5 \%$ & $47 \%$ & $3 \%$ & $4 \%$ \\
\hline
\end{tabular}

Fonte: Encuesta Nacional de Dinámica de Empleo e Innovación, ENDEI (2015).

A pesquisa contém diferentes tipos de informação sobre modernização tecnológica, como gastos, atividades, resultados relacionados à inovação e formalização do processo inovador, seja via departamentos específicos e alocação de trabalhadores diretamente ligados a estas atividades, seja via patentes dos resultados do processo.

A Tabela 1 mostra o percentual de empresas exportadoras em cada setor industrial e a distribuição do número de empresas, vendas e emprego por setor. A Tabela 2 apresenta as estatísticas descritivas (média e coeficiente de variação) das variáveis utilizadas nas estimações. Chama atenção a he- 
terogeneidade no grau de internacionalização das firmas argentinas, seja em relação à exportação (Tabela 1) ou à importação de insumos (Tabela 2).

Há dinamismo nas atividades exportadoras das indústrias farmacêutica, química e petroquímica, e de maquinaria e equipamentos, além de setores tradicionais de exportação argentina, como alimentos. ${ }^{4}$

Albornoz et al. (2012; 2014) mostram que o dinamismo das exportações argentinas justifica-se por quatro fenômenos simultâneos: o aumento do número de empresas com acesso a mercados externos, a ampliação na variedade de produtos em mercados já existentes, a abertura de novos mercados e o aumento dos volumes exportados. Em relação às características das empresas, predominam empresas pequenas, de propriedade familiar e que possuem, em média, metade dos trabalhadores não qualificados. Entretanto, o peso do porte é diferente por setor: em torno de $85 \%$ das empresas de alimentos e bebidas, química e petroquímica e automotiva são grandes, enquanto pequenas e médias empresas representam essa mesma proporção nos setores de madeira e móveis e metalurgia (ENDEI 2016).

A participação estrangeira no capital também varia substancialmente de acordo com o setor. Pouco mais de 93\% das empresas têm capitais nacionais, sendo que no complexo têxtil e de confecções representam 97\%. O setor farmacêutico, por outro lado, tem a maior participação de empresas com capitais estrangeiros, 17\% (ENDEI 2016). Em média, 25\% das empresas da amostra inovaram em produtos no período de análise. Observa-se certa associação entre inovação e acesso a novos mercados, dado que $42 \%$ das empresas que alcançaram resultados positivos a partir dos seus esforços de inovação também lograram ascender a novos mercados, seja internamente ou no exterior (apenas um terço destas empresas).

4 Segundo dados do comércio exterior do INDEC (indec.gob.ar), em 2012, os produtos das indústrias de alimentos representaram 18,75\% das exportações totais da Argentina. 
Tabela 2 - Penetração em mercados exportadores e determinantes

\begin{tabular}{|l|r|r|}
\hline \multicolumn{1}{|c|}{ Estatísticas Descritivas } & \multicolumn{1}{c|}{ Média } & \multicolumn{1}{c|}{$\begin{array}{c}\text { Coeficiente de } \\
\text { variação }\end{array}$} \\
\hline a) Variáveis de controle & & 0.0189 \\
\hline Tamanho: empresas pequenas & 0.3332 & 0.0288 \\
\hline Tamanho: empresas médias & 0.1072 & 0.0478 \\
\hline Tamanho: empresas grandes & 0.0451 & 0.0873 \\
\hline Participação capital internacional & 0.0621 & 0.0720 \\
\hline Forma parte de um grupo de empresas & 0.7689 & 0.0116 \\
\hline Empresa familiar & & \\
\hline b) Perfil inovador & 0.2522 & 0.0378 \\
\hline Inova em novos produtos & & \\
\hline c) Composição dos custos & 8.3532 & 0.0014 \\
\hline Log salário médio t-1 & 2.4279 & 0.0501 \\
\hline Proporção de gasto em investimento t-1 & 11.9456 & 0.0016 \\
\hline Log produtividade & & \\
\hline d: Composição da força de trabalho & 49.3601 & 0.0161 \\
\hline Proporção de operários não qualificados & 7.2709 & 0.0328 \\
\hline Proporção de técnicos & 6.4217 & 0.0331 \\
\hline Proporção de profissionais & & \\
\hline e: Participação em redes & & 0.1743 \\
\hline Cooperação com empresas do mesmo grupo & 0.3193 & 0.0321 \\
\hline Cooperação com outras firmas & 0.1845 & 0.0446 \\
\hline Cooperação com Universidades & 0.1867 & 0.0435 \\
\hline Cooperação com instituições públicas de P\&D & 0.3161 & 0.0318 \\
\hline Cooperação com Consultorias & 0.1002 & 0.0641 \\
\hline Cooperação através de Programas Públicos & & \\
\hline d: Provedores de insumos & 0.2055 & 0.0389 \\
\hline Mercado doméstico & 0.0450 & 0.0144 \\
\hline Mercosul-América Latina & 0.0756 & 0.0726 \\
\hline Ásia & & \\
\hline Europa - Estados Unidos & & \\
\hline
\end{tabular}

Fonte: Encuesta Nacional de Dinámica de Empleo e Innovación (2015).

Considerando a intensidade e os tipos de cooperação, a maior parte das empresas mantém relação com entidades de natureza privada, especialmente outras firmas e consultorias. Entre os motivos que promoveram a interação, destacam-se o desenvolvimento ou melhora de produtos/processos, gestão e/ou certificação de qualidade e capacitação de recursos 
humanos (ENDEI 2016). Em relação à composição da força de trabalho, predominam os trabalhadores em postos não qualificados, indicando a natureza majoritária de empresas produtoras de bens com baixo conteúdo tecnológico.

Na próxima seção são apresentados os resultados dos modelos que tratam das margens intensivas e extensivas da participação das empresas argentinas no comércio internacional, assim como dos fatores associados.

\section{Resultados}

A Tabela 3 apresenta os resultados dos modelos probit la e lb, que incorporam os determinantes clássicos da participação no comércio internacional (Dosi et al. 2015) e consideram as margens intensivas e extensivas do comércio. Isto se faz necessário para controlar a heterogeneidade das empresas e o possível viés de seleção no mercado internacional (Helpman et al. 2008). Além disso, contextualizam os modelos que incorporam os fatores tratados pela literatura, depois apresentados na Tabela 4. Em todos estes modelos, utiliza-se a mesma ponderação da amostra de empresas da ENDEI, de modo a respeitar os pesos de tamanho das empresas e setores nas estimativas.

Tabela 3 - Seleção em mercados exportadores

\begin{tabular}{|l|c|}
\hline \multicolumn{1}{|c|}{ Fatores associados à seleção } & Coeficiente \\
\hline Participação do capital internacional & $0,3377^{\star *}$ \\
$(\mathrm{D}=0$ sim; $\mathrm{D}=1$ não) & $(0,1422)$ \\
\hline Forma parte de um grupo de empresas & $0,1833^{\star}$ \\
$(\mathrm{D}=0$ sim; $\mathrm{D}=1$ não) & $(0,1142)$ \\
\hline Empresa familiar & $-0,0694$ \\
$(\mathrm{D}=0$ sim; $\mathrm{D}=1$ não) & $(0,0718)$ \\
\hline Log produtividade $\mathrm{t}-1$ & $\mathbf{0 , 4 0 6 2 ^ { \star \star * }}$ \\
\hline Wald chi ${ }^{2}$ & $\mathbf{( 0 , 0 2 5 1 )}$ \\
\hline Pseudo $\mathrm{R}^{2}$ & 639,46 \\
\hline
\end{tabular}

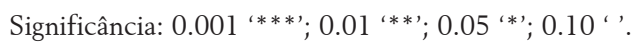


As variáveis de controle relativas às dummies de propriedade estrangeira e de afiliação a grupos apresentaram coeficientes marginalmente significativos, enquanto o coeficiente da dummy associada a controle familiar não se mostrou estatisticamente significativo. O Apêndice 1 expõe os resultados que incorporam também os diferentes setores industriais, que compreendem uma das principais fontes de heterogeneidade. Em relação à produtividade, a variável é significativa e positiva, indicando a possível existência de viés de seleção de empresas mais produtivas atuando nos mercados de exportação e a necessidade de se utilizar a razão inversa de Mills para atenuá-lo. Estima-se a razão inversa de Mills para empregá-la nos modelos probit ordenado (mercado de exportação por grau de desenvolvimento) e Poisson (número de produtos exportados), servindo de medida de probabilidade instantânea de deixar de ser parte da amostra.

A Tabela 4 apresenta os modelos que consideram a composição da força de trabalho, a interação da empresa em redes de cooperação em atividades de inovação e capacitação, entre outras, assim como a origem dos fornecedores. Os resultados indicam que empresas maiores (médias e grandes), com participação de capital internacional, que pertencem a um grupo econômico e que não são de propriedade familiar têm probabilidade maior de participar em mercados internacionais mais desenvolvidos. $\mathrm{O}$ coeficiente relativo ao tamanho da empresa é maior no modelo relacionado à diversidade de produtos, sugerindo a exigência de uma escala mínima para produzir competitivamente um número maior de bens.

Os coeficientes relativos à variável inovação em novos produtos são significativos e positivos, sendo maiores no modelo de número de produtos. Já no caso do investimento, utilizado como proxy de inovação em processos, o coeficiente é negativo, o que pode sugerir, dadas as características de predomínio de segmentos tradicionais e de baixa intensidade tecnológica da indústria argentina, que o investimento reflete mais reestruturação do que inovação em processos.

Nos dois modelos, os coeficientes para salários são positivos e significativos e sem diferenças consideráveis. Embora contraintuitivo à luz de trabalhos teóricos - em Melitz e Redding (2014) salários médios maiores representam maior custo e, consequentemente, menor competitividade - este resultado empírico é similar ao que Dosi et al. (2015) encontram para empresas exportadoras italianas, cujo custo por empregado era maior em quase todos os setores analisados. A explicação para o prêmio salarial pode 
ser o local de trabalho (empresa na qual o empregado trabalha), as habilidades ou maior produtividade dos trabalhadores (Card et al. 2013; Parrota et al. 2014; Garnero et al. 2014; Hanushek et al. 2015; Hospido 2015), ou o comércio internacional (Klein et al. 2013).

Tabela 4 - Fatores associados à penetração em mercados exportadores

\begin{tabular}{|c|c|c|c|c|}
\hline \multirow[b]{2}{*}{ Razão inversa de Mills } & \multicolumn{2}{|c|}{ Modelo destinos de exportação } & \multicolumn{2}{|c|}{ Modelo número de produtos } \\
\hline & $\begin{array}{c}-0.4774^{* * *} \\
(0.1159)\end{array}$ & $\begin{array}{l}-0.2343^{* *} \\
(0.1168)\end{array}$ & $\begin{array}{c}-1.6717^{* * *} \\
(0.1720)\end{array}$ & $\begin{array}{c}-1.3428^{* * *} \\
(0.1785)\end{array}$ \\
\hline \multicolumn{5}{|l|}{ H2: Características da empresa } \\
\hline Tamanho: média (H2) & $\begin{array}{l}0.2403^{\text {*** }} \\
(0.0669)\end{array}$ & $\begin{array}{l}0.1751^{* * *} \\
(0.0685)\end{array}$ & $\begin{array}{l}0.6142^{* * *} \\
(0.1248)\end{array}$ & $\begin{array}{l}0.5241^{* \star *} \\
(0.1252)\end{array}$ \\
\hline Tamanho: grande $(\mathrm{H} 2)$ & $\begin{array}{l}0.5173^{* * *} \\
(0.1067)\end{array}$ & $\begin{array}{l}0.3416^{* * *} \\
(0.1071)\end{array}$ & $\begin{array}{l}1.1335^{* * *} \\
(0.1513)\end{array}$ & $\begin{array}{l}0.9860^{* * *} \\
(0.1477)\end{array}$ \\
\hline \multicolumn{5}{|l|}{ H3: Inovação } \\
\hline Inova: novos produtos $(\mathrm{H} 3)$ & $\begin{array}{l}0.3217^{* * *} \\
(0.0625)\end{array}$ & $\begin{array}{l}0.2221^{* * *} \\
(0.0654)\end{array}$ & $\begin{array}{l}0.5599^{* * *} \\
(0.1133)\end{array}$ & $\begin{array}{l}0.4049^{\star * *} \\
(0.1097)\end{array}$ \\
\hline Inova: novos processos $(\mathrm{H} 3)$ & $\begin{array}{l}-0.0658^{* *} \\
(0.0248)\end{array}$ & $\begin{array}{c}-0.0325 \\
(0.0259)\end{array}$ & $\begin{array}{l}-0.0243 \\
(0.0361)\end{array}$ & $\begin{array}{l}-0.0086^{* * *} \\
(0.0342)\end{array}$ \\
\hline \multicolumn{5}{|l|}{ H4: Custos } \\
\hline Log salário médio t-1 (H4) & $\begin{array}{l}0.2960^{* * *} \\
(0.0591)\end{array}$ & $\begin{array}{l}0.2343^{* * *} \\
(0.0587)\end{array}$ & $\begin{array}{l}0.3096^{* * *} \\
(0.0804)\end{array}$ & $\begin{array}{l}0.2975^{* * *} \\
(0.0839)\end{array}$ \\
\hline \multicolumn{5}{|l|}{ H5: Composição mão de obra } \\
\hline Proporção de operários não qualificados & $\begin{array}{c}0.0003 \\
(0.0009) \\
\end{array}$ & $\begin{array}{c}0.0005 \\
(0.0009) \\
\end{array}$ & $\begin{array}{c}0.0018 \\
(0.0015)\end{array}$ & $\begin{array}{c}0.0020 \\
(0.0014) \\
\end{array}$ \\
\hline Proporção de técnicos & $\begin{array}{l}0.0084^{* * *} \\
(0.0020)\end{array}$ & $\begin{array}{l}0.0066^{* * *} \\
(0.0021)\end{array}$ & $\begin{array}{l}0.0568 \\
(0.0023)\end{array}$ & $\begin{array}{l}0.0053^{* *} \\
(0.0023)\end{array}$ \\
\hline Proporção de profissionais & $\begin{array}{l}0.0144^{* * *} \\
(0.0029)\end{array}$ & $\begin{array}{l}0.0120^{* * *} \\
(0.0029)\end{array}$ & $\begin{array}{l}0.0110^{* * *} \\
(0.0024)\end{array}$ & $\begin{array}{l}0.0114^{* * *} \\
(0.0026)\end{array}$ \\
\hline \multicolumn{5}{|l|}{ H6: Participação em redes } \\
\hline Cooperação: empresas do mesmo grupo & - & $\begin{array}{r}-0.1348 \\
(0.1060) \\
\end{array}$ & - & $\begin{array}{c}-0.2725^{\mathrm{xx}} \\
(0.099)\end{array}$ \\
\hline Cooperação: outras firmas & - & $\begin{array}{l}0.1427^{1} \\
(0.0617)\end{array}$ & - & $\begin{array}{c}0.0315 \\
(0.0805) \\
\end{array}$ \\
\hline Cooperação: Universidades & - & $\begin{array}{l}0.1970 \\
(0.0913)\end{array}$ & - & $\begin{array}{l}0.2101 \\
(0.0899)\end{array}$ \\
\hline Cooperação: instituições públicas de C\&T & - & $\begin{array}{l}0.1813^{2} \\
(0.0739)\end{array}$ & - & $\begin{array}{l}0.2023 \\
(0.0863)\end{array}$ \\
\hline Cooperação: Consultoras & - & $\begin{array}{c}0.0236 \\
(0.0631) \\
\end{array}$ & - & $\begin{array}{c}0.0561 \\
(0.0842)\end{array}$ \\
\hline Cooperação: Programas Públicos & - & $\begin{array}{c}0.1215 \\
(0.1297) \\
\end{array}$ & - & $\begin{array}{l}-0.2030 \\
(0.1353) \\
\end{array}$ \\
\hline \multicolumn{5}{|l|}{ H7: Fornecedores de insumos } \\
\hline Tipo I: Mercosul-América Latina & - & $\begin{array}{l}0.7792^{* * *} \\
(0.1021)\end{array}$ & - & $\begin{array}{c}0.6167^{* * *} \\
(0.151)\end{array}$ \\
\hline Tipo II: Asia & - & $\begin{array}{l}0.8285^{* * *} \\
(0.0895)\end{array}$ & - & $\begin{array}{l}1.2131^{* * *} \\
(0.1468)\end{array}$ \\
\hline Tipo III: Europa - Estados Unidos & - & $\begin{array}{l}0.9258^{* * *} \\
(0.0735)\end{array}$ & - & $\begin{array}{l}0.8772^{\text {*** }} \\
(0.1155)\end{array}$ \\
\hline Wald & 701,44 & 1028,79 & 1644,08 & 2130,56 \\
\hline Pseudo R ${ }^{2}$ & 0.1504 & 0.2074 & - & - \\
\hline Observações & 3261 & 3261 & 3261 & 3261 \\
\hline
\end{tabular}

Significância: ${ }^{* * *} \mathrm{p}<0.01,{ }^{* *} \mathrm{p}<0.05,{ }^{*} \mathrm{p}<0.1$ 
No caso específico da Argentina, Bustos (2011), com dados de um painel de 1600 firmas industriais, ${ }^{5}$ mostra que as empresas exportadoras têm maior nível tecnológico e são mais intensivas em trabalho qualificado. $\mathrm{O}$ comércio internacional produz dois tipos de realocação do trabalho: a) via mudança estrutural, com os trabalhadores indo para setores ou empresas mais intensivas em habilidades (indústrias de maior conteúdo de conhecimento ou tecnologia); b) via aumento na demanda por trabalho qualificado, independente do componente setorial e relacionando-se mais à própria atividade exportadora.

Nas estimações do prêmio salarial, consideram-se três grupos de trabalhadores: operários não qualificados, técnicos (operários qualificados) e profissionais. O coeficiente para o primeiro grupo, que tenta captar a influência de trabalhadores de baixo custo na exportação, mostrou-se positivo mas não significativo nos dois modelos, indicando que o custo salarial de trabalhadores sem qualificação nas empresas argentinas autosselecionadas para o comércio internacional não afeta a participação no mercado externo. Já os coeficientes para os grupos que representam trabalhadores com habilidades específicas são positivos e significativos em ambos os modelos, sugerindo spillovers de conhecimento gerados pela exportação a mercados mais desenvolvidos (Parrotta et al. 2014). Desta forma, os resultados mostram a interação entre diversidade da mão de obra e inserção da empresa em mercados com diferentes graus de desenvolvimento.

A diversidade da força de trabalho associa-se com a diversidade na organização da produção e na tecnologia utilizada. Indústrias tradicionais, caracterizadas por atividades mais rotineiras, requerem uma força de trabalho mais homogênea, enquanto setores intensivos em conhecimento, expostos à concorrência internacional e à mudança tecnológica, demandam maior heterogeneidade das atividades e habilidades (Biscourp e Kramarz 2007; Serti e Tomasi 2014; Iodice e Tomasi 2015). Os coeficientes que aumentam com as habilidades dos trabalhadores captam o fato de que, para aumentar a probabilidade de se posicionar comercialmente em países mais desenvolvidos, as empresas dependem de uma força de trabalho mais heterogênea e baseada em profissionalização, criatividade e inovação. Barletta et al. (2014) apresentam evidências empíricas de que a maior capacitação dos trabalhadores eleva a produtividade e a competitividade das empresas argentinas.

5 Pesquisa Nacional de Inovação e Conduta Tecnológica das Empresas Argentinas (ENIT-INDEC), para o período 1992-1996. 
Os modelos 2 e 4 incluem variáveis relacionadas à cooperação em redes, como capacitação de recursos humanos, pesquisa e desenvolvimento, intercâmbio tecnológico, gestão de qualidade e atividades de desenho industrial. O resultado da estimação para colaboração com empresas do mesmo grupo ou subsidiárias é negativo e significativo apenas no modelo 4. Os indicadores de cooperação horizontal (empresas de outros grupos) e a jusante (universidades e instituições públicas de C\&T) são todos positivos e significativos. Em contraste com Alvarez et al. (2009), o resultado do modelo que considerou o número de produtos exportados indica que as redes internas contribuem negativamente, sendo aproveitadas apenas as fontes externas de habilidades complementares. No caso específico das firmas argentinas, os efeitos positivos acontecem na interação com universidades e instituições públicas de pesquisa e desenvolvimento, e em menor grau, com firmas de outros grupos e consultorias privadas.

Este resultado revela a importância da cooperação para melhorar a posição nos mercados, fato reforçado pelas características próprias do processo de participação no comércio de empresas deste tipo de país. A cooperação permite compartilhar a natureza incerta e dispendiosa da pesquisa, do acesso aos mercados e da procura de oportunidades (Alvarez et al. 2009; Vaccaro et al. 2010), mas há também a necessidade de estruturar eficientemente a cadeia de produção para melhorar o desempenho (Bernard et al. 2015). A conformação destas redes capta a essência da ideia de interdependência sistêmica, na qual o resultado do comportamento de cada agente é estritamente dependente das interações dentro de um sistema (Antonelli 2008).

Os resultados mostram que a probabilidade de penetrar em mercados mais desenvolvidos cresce com a quantidade e a diversidade das fontes dos fornecedores de insumos intermediários. Entretanto, os parâmetros diferem segundo a variável dependente: no modelo cuja variável dependente refere-se aos destinos de exportação, o maior coeficiente se relaciona a fornecedores da Europa e Estados Unidos, enquanto no modelo que estima o número de bens exportados, o maior coeficiente corresponde à Ásia. Este resultado pode ser interpretado à luz dos possíveis canais entre insumos intermediários e desempenho. O primeiro canal atua via custos: a queda dos preços relativos afeta a lucratividade ou permite concorrer por preços. Os insumos adquiridos no MERCOSUL e em países da América Latina representam este canal, uma vez que se trata de nações com características tecnológicas similares à Argentina. O segundo mecanismo é a variedade, 
que permite expandir o conjunto de insumos. Neste caso, os três grupos de países considerados afetam positivamente a variedade, melhorando as decisões de exportação. O canal relacionado ao upgrading de qualidade e tecnologia está representado pelos insumos provenientes da Europa, Estados Unidos e alguns países de Ásia. A magnitude dos coeficientes evidencia que este último canal é o mais importante para explicar o acesso a mercados mais desenvolvidos.

Este efeito, combinado com os resultados sobre tamanho e internacionalização da propriedade, pode ser analisado pelo papel dos países em desenvolvimento, detentores de recursos naturais e produtos primários, nas cadeias globais de valor (CGV) (Gereffi 2014). Quase 50\% da amostra analisada (e da estrutura produtiva argentina) é composta por indústrias manufatureiras baseadas em recursos naturais, como alimentos e bebidas, couro e madeira. Nestes setores, existe uma mudança na organização das CGVs caracterizada pelo aumento do poder de produtores em economias emergentes, que tem a Argentina como um participante importante. Neste contexto, os fornecedores especializados e redes de cooperação em serviços (desenho, pesquisa e desenvolvimento, e gestão de qualidade) têm um papel fundamental para a inserção das empresas argentinas em mercados mais desenvolvidos, o que está de acordo com os resultados encontrados. Especificamente o setor de alimentos, que representa quase 30\% da indústria de transformação argentina, atraiu grandes firmas globais, que via aquisição de capital de empresas ou colaboração estratégica buscavam assegurar a oferta sustentável de matérias-primas (Pietrobelli e Staritz 2013; Gereffi 2014).

Por fim, cabe destacar que a razão inversa de Mills é estatisticamente significativa em todos os modelos, justificando a sua inclusão para atenuar o viés de seleção. Isto é, a restrição de exclusão em mercados exportadores (produtividade) é uma forte barreira à entrada, que se omitida no exercício empírico levaria à superestimação dos coeficientes estimados. 


\section{Conclusões}

Este trabalho analisou o acesso de empresas da indústria de transformação da Argentina, país em desenvolvimento tecnologicamente distante da fronteira, a mercados externos com diferentes graus de desenvolvimento. Dado este objetivo, foram testadas algumas hipóteses, considerando um modelo que leva em conta a participação das empresas no comércio internacional (margem extensiva) e um modelo focalizando também o tipo e qualidade desta inserção (margem intensiva).

Algumas características das empresas revelaram-se importantes no tipo de inserção externa: tamanho, participação de estrangeiros na propriedade do capital e propriedade não familiar aumentam a probabilidade de que a empresa exporte mais ou exporte para mercados mais desenvolvidos. Ademais, empresas que inovam tendem a exportar para mercados mais complexos, uma vez que estreitam a brecha tecnológica e se tornam competitivas, superando as deficiências do setor ou do país.

Para as empresas da amostra da ENDEI, empresas que exportam para mercados de maior complexidade apresentam produção mais intensiva em tecnologia e em trabalho qualificado, resultado consistente com a importância das habilidades técnicas e profissionais dos empregados para penetrar tais mercados. Por fim, as relações de cooperação da empresa com instituições mostraram-se importantes na exportação a mercados mais complexos e na importação de insumos que afetam a inserção internacional. Isto decorre da redução de custos ou do acesso a uma maior variedade de insumos ou a novas tecnologias. Estes resultados são cruciais em tempos de aprofundamento das cadeias globais de valor e da inserção das empresas de um país como Argentina. 


\section{Referências}

Acemoglu, Daron, Pol Antràs, e Elhanan Helpman. 2007. "Contracts and Technology Adoption.” American Economic Review 97(3): 916-943, June .

Aiginger, Karl, Susanne Bärenthaler-Sieber e Johanna Vogel. 2015. "Competitiveness under New Perspectives." Working Paper 44, WWW For Europe.

Albornoz F., Calvo P., Coremberg A., Heymann D. e Alejandro Vicondoa. 2012. "Patrones de acumulación, comercio exterior y evolución industrial en la Argentina". Boletín Informativo Techint 339 (Septiembre-Diciembre).

Albornoz, Facundo, Ezequiel García Lembergman, e Leticia Juarez. 2014. "Dimensiones microeconómicas de un boom exportador". Anais da XLIX Reunión Anual AAEP.

Alvarez, Isabel, Raquel Marin e Antonio Fonfría. 2009. "The role of networking in the competitiveness of firms." Technological Forecasting \& Social Change 76: 401-421, March.

Amable, Bruno, Bart Verspagen. 1995. "The role of technology in market shares dynamics." Applied Economics 27:197-204.

Amendola, Giovanni, Giovanni Dosi, e Erasmo Papagni. 1993. "The dynamics of international competitiveness". Weltwirtschaftliches Archiv 129(129): 451-471, September.

Antonelli, Cristiano. 2008. "Localised Technological Change". New York: Routledge.

Antràs, Pol, Teresa Fort, e Felix Tintelnot. 2017. "The Margins of global Sourcing: Theory and Evidence from U.S. Firms.” American Economic Review 107(9): 2514-64, September.

Artopoulos, Alejandro, Daniel Friel e Juan Carlos Hallak. 2013. "Export emergence of differentiated goods from developing countries: Export pioneers and business practices in Argentina." Journal of Development Economics 105: 19-35, November.

Aw, Bee Yan, Mark Roberts e Tor Winston. 2007. "Export Market Participation, Investments in R\&D and Worker Training, and the Evolution of Firm Productivity." The World Economy 30(1): 83-104, January.

Bastos, Paulo e Joana Silva. 2010. "The quality of a firm's exports: where you export to matters." Journal of International Economics 82(2): 99-111, November.

Beise-Zee, Rian e Christian Rammer. 2006. "Local User-Producer Interaction in Innovation and Export Performance of Firms." Small Business Economics 27(2-3): 207-222, October.

Bernard, Andrew, Bradford Jensen, Stephen Redding e Peter Schott. 2012. "The empirics of firm heterogeneity and international trade." Annual Review of Economics 4:283-313, September.

Bernard, Andrew, Andreas Moxnes e Yukiko Saito. 2015. "Production Network, Geography and Firm Performance.” NBER Working Paper 21082, April.

Biscourp, Pierre e Francis Kramarz. 2007. "Employment, skill structure and international trade: Firm-level evidence for France." Journal of International Economics 72(1): 22-51, May.

Bustos, Paula. 2011. "Trade Liberalization, Exports, and Technology Upgrading: Evidence on the Impact of Mercosur on Argentinian Firms." American Economic Review 101(1): 304-40, February.

Card, David, Jörg Heining, e Patrick Kline. 2013. "Workplace Heterogeneity and the Rise of West German Wage Inequality." Quarterly Journal of Economics, vol. 128(3): 967-1015, August.

Carluccio, Juan, Denis Fougère, e Erwan Gautier. 2015. "Trade, wages, and collective bargaining: Evidence from France.” Economic Journal 125(584): 803-837, May.

Dosi, Giovanni, Marco Grazzi e Daniele Moschella. 2015. "Technology and cost in international competitiveness: From countries and sectors to firms.” Research Policy 44(10): 1795-1814.

Dunning, John. 2016. "Location and the Multinational Enterprise: A Neglected Factor?” In Location of International Busi (ed.) Location of International Business Activities. (Basingstoke: Palgrave McMillan,). 
ENDEI, Ministerio de Ciencia, Tecnologia e Innovación Productiva Encuesta Nacional de Empleo e Innovación: documento de difusión. 2016. Acessado em: http://indicadorescti.mincyt.gob.ar.

Fieler, Ana Cecília, Marcela Eslava e Daniel Xu. 2014. “Trade, Skills and Quality Upgrading: A Theory with Evidence from Colombia.” NBER Working Paper 19992, March.

Garnero, Andrea, Kampelmann, Stephan e François Rycx. 2014. "The heterogeneous effects of workforce diversity on productivity, wages, and profits.” Industrial Relations 53(3): 430-477, June.

Gereffi, Gary. 2014. "Global value chains in a post-Washington Consensus world." Review of International Political Economy 21(1): 9-37.

Greene, William e David Hensher. 2010. "Modeling ordered choices: a primer”. Cambridge: Cambridge University Press.

Guerreri, Paolo e Valentina Meliciani. 2005. "Technology and international competitiveness: The interdependence between manufacturing and producer services." Structural Change and Economic Dynamics 16(4): 489-502.

Hallak, Juan Carlos. 2006. "Product quality and the direction of trade." Journal of International Economics 68(1): 238-265.

Helpman, Elhanan, Oleg Itskhoki, Marc-Andreas Muendler e Stephen Redding. 2017. "Trade and Inequality: from Theory to Estimation." Review of Economic Studies 84: 357-405.

Helpman, Elhanan, Marc Melitz e Yona Rubinstein. 2008. "Estimating trade flows: trading partners and trading volumes.” The Quarterly Journal of Economics 123(2): 441-487, May.

Hospido, Laura. 2015. "Wage dynamics in the presence of unobserved individual and job heterogeneity." Labour Economics 33: 81-93, April.

Iodice, Irene e Chiara Tomasi. 2015. "Skill upgrading, wage gap and international trade: Firm-level evidence for Italian manufacturing firms.” LEM Working Paper Series, N.2015/06.

Itskhoki, O.; Helpman. E. 2015. "Trade Liberalization and Labor Market Dynamics with heterogeneous firms”. Princeton Papers.

Klein, Michael, Christoph Moser e Dieter Urban. 2013. "Exporting, skills and wage inequality.” Labour Economics 25: 76-85, December.

Laursen, Keld e Valentina Meliciani. 2002. "The relative importance of international vis-a-vis national technological spillovers for market share dynamics." Industrial and Corporate Change 11: 875-894.

Laursen, Keld e Valentina Meliciani. 2010. "The role of ICT knowledge flows for international Market share dynamics.” Research Policy 39(5): 687-697, June.

Melitz, Marc e Stephen Redding. 2014. "Heterogeneous Firms and Trade.” Handbook of International Economics 4: 1-54.

Parrotta, Pierpaolo, Dario Pozzoli e Mariola Pytlikova. 2014. "Labor diversity and firm productivity.” European Economic Review 66: 144-179, February.

Pietrobelli, Carlo e Cornelia Staritz. 2013. "Challenges for Global Value Chain Interventions in Latin America." Technical Note N. IDB-TN-548. Inter-American Development Bank.

Serti, Francesco e Chiara Tomasi. 2014. "Export and import market-specific characteristics." Empirical Economics 47(4): 1467-1496, December.

Vaccaro, Antonio, Ronaldo Parente e Francisco Veloso. 2010. "Knowledge Management Tools, Inter-Organizational Relationshps, Inovation and Firm Perfomance." Technological Forecasting and Social Change 77(7): 1076-1089, September. 


\section{Apêndice 1 - Coeficientes estimados para os setores industriais}

\begin{tabular}{|l|r|r|}
\hline \multicolumn{1}{|c|}{ Setor industrial (categoria de referência: Alimentos) } & \multicolumn{1}{c|}{ Coeficiente } & Erro Padrão \\
\hline Artigos têxteis e vestuário & 0,0372 & 0,0654 \\
\hline Preparação e fabricação de artefatos de couro & $-0,6081$ & 0,0845 \\
\hline Fabricação de produtos de madeira & $-0,1966$ & 0,0783 \\
\hline Fabricação de celulose, papel e produtos de papel & 0,1083 & 0,0579 \\
\hline Edição & 0,3379 & 0,0535 \\
\hline Produtos químicos & 0,1607 & 0,0498 \\
\hline Produtos de borracha e plástico & $-0,2160$ & 0,0690 \\
\hline Outros minerais não metálicos & $-0,1049$ & 0,0769 \\
\hline Metais comuns & 0,2185 & 0,0431 \\
\hline Outros produtos de metal & 0,4807 & 0,0730 \\
\hline Maquinaria e equipamentos & 0,6834 & 0,0912 \\
\hline Instrumentos médicos & $-0,0680$ & 0,1156 \\
\hline Outros equipamentos de transportes & 0,2087 & 0,0608 \\
\hline Móveis & 0,7031 & 0,0624 \\
\hline Máquina ferramentas em geral & $-0,2503$ & 0,0745 \\
\hline Frigoríficos & $-0,4503$ & 0,1042 \\
\hline Produtos lácteos & 1,4025 & 0,0889 \\
\hline Vinhos e outras bebidas fermentadas & 0,3837 & 0,0788 \\
\hline Farmacêuticas & 0,7083 & 0,0940 \\
\hline Maquinaria Agropecuária e Florestal & 0,1527 & 0,0977 \\
\hline Aparelhos de uso doméstico & 0,1479 & 0,0636 \\
\hline Material elétrico, rádio e televisão & $-0,6440$ & 0,1517 \\
\hline Fabricação e montagem de veículos automotores, reboques e carrocerias & 0,3454 & 0,0626 \\
\hline Autopeças & 0,5554 & 0,1088 \\
\hline & & \\
\hline
\end{tabular}

Fonte: Estimações com base em ENDEI. 